\title{
Mass Loss, Kinematics, \& Evolution of Super Star Clusters in the Antennae
}

\author{
Andrea M. Gilbert \\ Max-Planck Institut für extraterrestrische Physik, Postfach 1312, \\ D-85748 Garching, Germany
}

James R. Graham

University of California, Berkeley, CA 94720-3411, USA

\begin{abstract}
The youngest super star clusters (SSCs) in the merging Antennae Galaxies (NGC 4038/39) drive supersonic mass-loaded outflows from the HII regions in which they are embedded. High-resolution K-band Keck/NIRSPEC spectroscopy reveals broad, spatially extended $\operatorname{Br} \gamma$ emission in 16 targets. Simple wind models for the line profiles provide good fits and imply cluster mass-loss rates of up to $1.5 \mathrm{M}_{\odot} /$ year and terminal velocities of up to $205 \mathrm{~km} \mathrm{~s}^{-1}$. The emission-line clusters (ELCs) that drive these outflows constitute at least $15 \%$ of the star formation rate in the Antennae, and their high star formation efficiencies imply that they will probably evolve into bound SSCs. The youngest ELC outflows, which are driven primarily by stellar winds, very efficiently entrain ambient matter. The cluster winds transfer or dissipate a large fraction of their energy and momentum in a hot or cool medium that does not emit Br $\gamma$. ELCs are the individual engines that power galactic-scale superwinds, viewed in their earliest evolutionary stage.
\end{abstract}

\section{Emission-Line Clusters in the Antennae}

Star formation in starbursts creates massive young super star clusters (SSCs) that are not often found in more quiescent environments. Their high masses and stellar densities resemble those of globular clusters (GCs), though their ages are measured in Myr rather than Gyr. This suggests an evolutionary sequence in massive star-forming regions: GCs may evolve from SSCs, whose precursors are ultra-dense compact $\mathrm{H}$ II regions detected in radio surveys (e.g. Johnson et al. 2003 and references therein). In order to study their nebular kinematics, we conducted a K-band spectroscopic survey of a sample of the youngest SSCs in the nearby merger NGC 4038/39, the Antennae Galaxies, whose large SSC population is well-studied (e.g. Whitmore \& Zhang 2002). We discovered broad, supersonic Br $\gamma$ lines in Keck/NIRSPEC echelle spectra of 16 of 17 targets (Gilbert 2002). The Br $\gamma$ profiles are well resolved (spectral resolution was $12 \mathrm{~km} \mathrm{~s}^{-1}$ ), have widths of $60-105 \mathrm{~km} \mathrm{~s}^{-1}$ FWHM, and exhibit high-velocity non-Gaussian wings (Fig. 1) We refer to these broad-line SSCs as emission-line clusters (ELCs). Turner et al. (2003) also found an ELC in NGC 5253.

Giant extra-galactic $\mathbf{H}$ II regions (GHIIRs) are characterized by supersonic line widths as large as the lowest ELC widths (Smith \& Weedman 1970) as well as large sizes $(d>100 \mathrm{pc})$ and Lyman continuum fluxes of order $\mathrm{Q}=10^{51}$ $\mathrm{s}^{-1}$. ELCs are typically more compact $(d \sim 40-80 \mathrm{pc})$ and brighter, with $\mathrm{Q}$ $=10^{51}-10^{53} \mathrm{~s}^{-1}$. GHIIR recombination line fluxes are positively correlated 

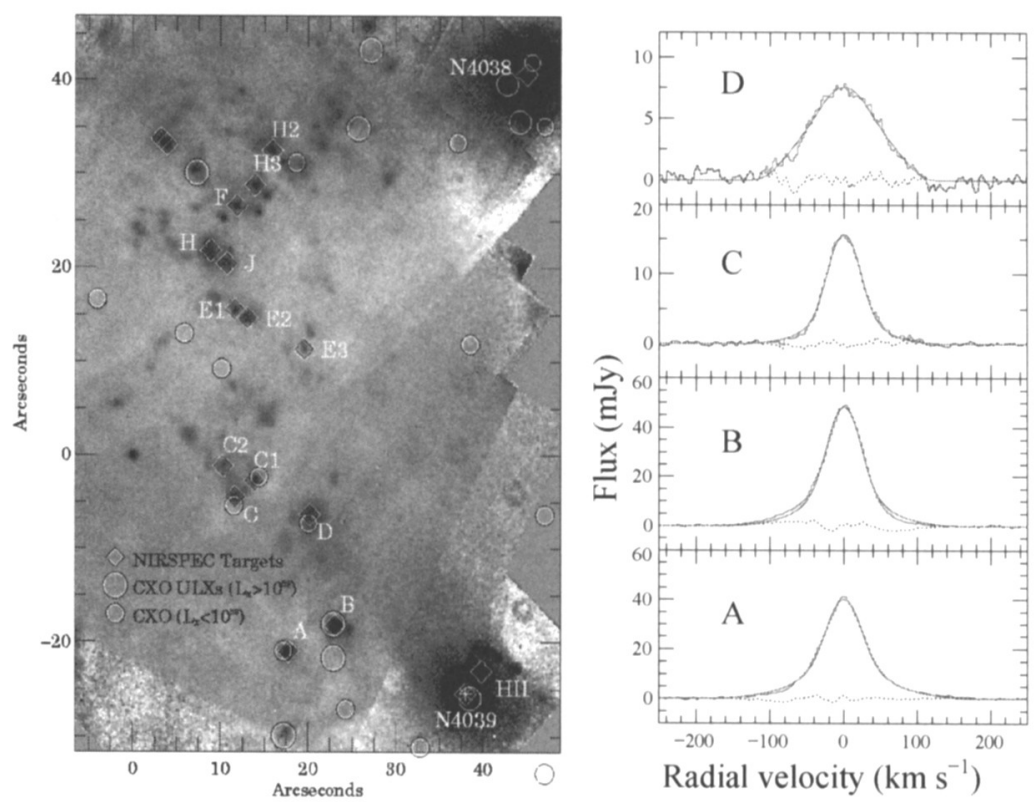

Figure 1. Left: K-band mosaic of the central region of the Antennae identifies ELCs and positions of Chandra sources (Zezas et al. 2002). Right: Supersonic Br $\gamma$ profiles of four ELCs with over-plotted wind models and fit residuals.

with their line widths (e.g. Fuentes-Masip et al. 2000), and ELCs display the same correlation, extending it to larger line fluxes and widths (Gilbert 2002). GHIIRs and ELCs excite similar quantities of gas $\left(10^{5}-10^{6} \mathrm{M}_{\odot}\right)$ but the mean emissivity-weighted gas density in an ELC is several orders of magnitude higher than that in GHIIRs (Gilbert 2002). This may be due to higher pressure in the interstellar medium (ISM) of a merger than in a typical galactic disk.

$\mathrm{Br} \gamma$ line fluxes and $\mathrm{K}$ magnitudes imply ELC ages of 3-8 Myr and masses of $10^{5}-10^{7} \mathrm{M}_{\odot}$ with the help of Starburst99 models (Leitherer et al. 1999). IR spectroscopy and radio recombination line fluxes yield $A_{K}=0.2-1.1 \mathrm{mag}$ for some ELCs (Gilbert 2002 and references therein). Comparing the virial line widths (from ELC masses and sizes) with observed ones suggests that much of the gas in ELC outflows is unbound.

A massive stellar cluster produces X-rays due to colliding stellar winds and $\mathrm{X}$-ray binaries (XRBs). A model for the X-ray emission of an ELC produces values comparable with Chandra fluxes near Eddington for ELCs with likely Xray counterparts (Fig. 1; Zezas et al. 2002), but not for the ULX that appears coincident with ELC B and thus must be a more exotic object, e.g. beamed $\mathrm{XRB}$ or intermediate-mass black hole (Gilbert 2002).

\section{Mass-Loaded Outflows from Emission-Line Clusters}

In order to determine the mass-loss rates of ELCs, we model their supersonic $\operatorname{Br} \gamma$ profiles with a semi-empirical $\beta$-wind law. This velocity law is predicted and 

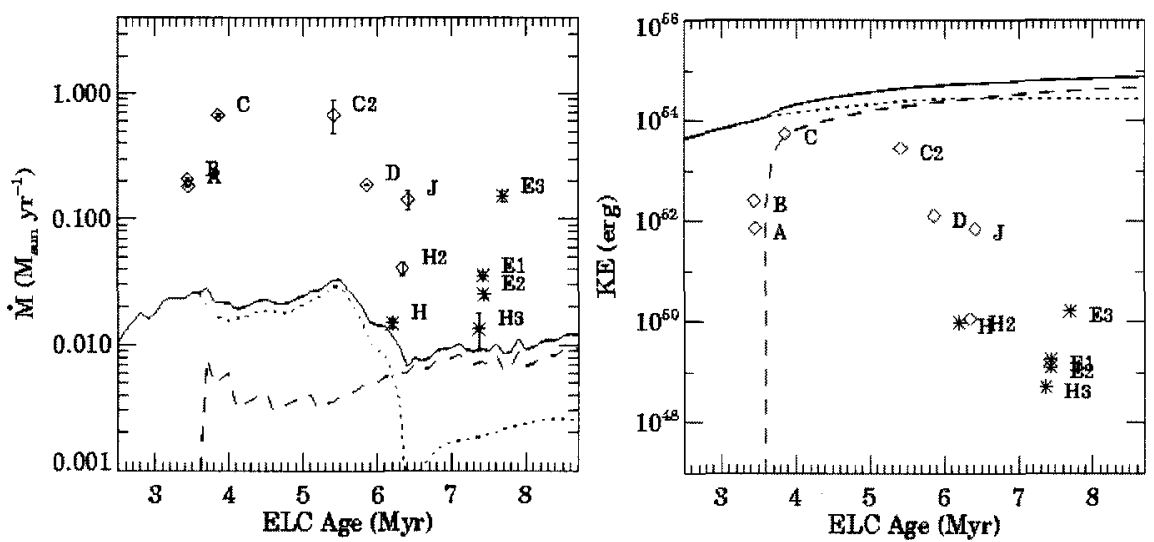

Figure 2. Mass-loss rates (left) and kinetic energy (right) measured for ELC outflows (points, normalized to a $10^{6} \mathrm{M}_{\odot}$ cluster). Curves are predictions from Starburst99 models (Leitherer et al. 1999) for a cluster's stellar winds (dotted), SNe (dashed), and their sum (solid). ELC outflows display much higher $\dot{M}_{\mathrm{HII}}$ and lower KEs than models predict, suggesting efficient mass loading and thermalization of $\mathrm{KE}$.

observed for radiation-driven wind lines of hot stars (Kudritzki \& Puls 2000), but we find that it also provides an excellent semi-empirical model for our data. We parameterized the line profile models with six unknowns: inner and outer wind radii $r_{0}$ and $r_{1}$, mass-loss rate $\dot{M}_{\mathrm{HII}}$, the power-law slope $\beta$ for the velocity law $v(r)=v_{\infty}\left(1-b \frac{r_{0}}{r}\right)^{\beta}$ where $b \equiv 1-\left[v\left(r_{0}\right) / v_{\infty}\right]^{\frac{1}{\beta}}$, adopted for the lineemitting gas, and the terminal velocity of the wind $v_{\infty}$. The Doppler broadening parameter of the gas for a Gaussian line profile, $v_{D}=\sqrt{2} \sigma=\sqrt{2 k T / \mu}=16.3$ $\mathrm{km} \mathrm{s}^{-1}$, is fixed at that of a $10^{4} \mathrm{~K}$ plasma. We fixed the wind velocity at $r_{0}$, $v_{0}$, equal to $v_{D}$, since it tends to be comparable with $v_{D}$ and has little effect on the profile (Kudritzki \& Puls 2000). Assuming a constant mass-loss rate $\left(\dot{M}_{\mathrm{HII}}=4 \pi r^{2} \rho v\right)$, the velocity law and $\dot{M}_{\mathrm{HII}}$ prescribe a density law $\rho(r)$ for the emitting gas. With this recipe we can compute the $\mathrm{Br} \gamma$ flux from the flow as a function of radial velocity, and compare the resulting line profile with the observed one. The predicted line profiles have broad non-Gaussian wings and provide excellent fits to the observed line profiles (Fig. 1).

The mass loss rates values derived from the $\beta$ model range over $0.006-1.5$ $\mathrm{M}_{\odot} \mathrm{yr}^{-1}$. The most powerful ELC outflow has an observed mass-loss rate that is equivalent to the combined winds of about $10^{6} \mathrm{O}$ stars or $10^{4} \mathrm{WR}$ stars. At an age of $3 \mathrm{Myr}$ a $10^{6} \mathrm{M}_{\odot}$ cluster has about $350 \mathrm{WR}$ stars and $4500 \mathrm{O}$ stars for a Salpeter IMF (1-100 $\mathrm{M}_{\odot}$, Starburst99 model), which provide a total mass-loss rate in wind ejecta that is nearly two orders of magnitude lower than inferred from our $\mathrm{Br} \gamma$ profile models. We detect much more out-flowing photoionized gas than is ejected directly from stars; thus ELC outflows efficiently entrain matter. This effect is also observed in starburst superwinds (e.g. NGC 1569, Martin, Kobulnicky, \& Heckman 2002), which are powered by the joint energy input from many clusters into the ISM. 
The terminal velocities of ELC outflows range from a few times the sound speed up to $200 \mathrm{~km} \mathrm{~s}^{-1}$, significantly less than terminal velocity of a single $\mathrm{O}$ star wind, a few $1000 \mathrm{~km} \mathrm{~s}^{-1}$. This provides another indication that we are not directly observing mass loss from hot stars. The low terminal velocities may be due to the interaction in close quarters of many individual winds, photoevaporative flows, (in older clusters) supernova explosions, and ultimately the collision with ambient neutral gas that the outflow encounters and entrains.

The discrepancy between observed $\dot{M}_{\mathrm{HII}}$ and predicted stellar mass loss due to winds and supernovae ( $\mathrm{SNe}$ ) decreases with increasing cluster age, shown in Fig. 2 for all ELCs (normalized to a mass of $10^{6} \mathrm{M}_{\odot}$ ). Either older ELCs entrain matter less efficiently, or they have swept away most of their reservoir of neutral gas. Fig. 2 also displays the measured and predicted kinetic energies (KEs) of the outflows. In $\mathrm{Br} \gamma$ we detect only a small fraction of the $\mathrm{KE}$ available from winds and SNe; the rest must reside in a hotter and/or cooler medium. Stellar wind shocks generate X-rays, and Chandra observations reveal possible counterparts for some ELCs (e.g. Zezas et al. 2002). Efficient thermalization of mechanical energy in a starburst is a necessary condition for generating a large-scale superwind, the earliest phase of which may be taking place in the Antennae.

The star-formation rate (SFR) in our small sample of ELCs is $4 \mathrm{M}_{\odot} \mathrm{yr}^{-1}$ (nearly half of this in a single ELC), so ELCs comprise at least $15 \%$ of the total SFR in the Antennae. A simple estimate of ELC star-formation efficiencies gives values of 30-70\%, suggesting that they stand a good chance of becoming bound SSCs, which in turn may evolve into GCs (Gilbert 2002).

Acknowledgments. We thank the Hawaiian community for the opportunity to observe on Mauna Kea and the staff of the Keck Observatory for assistance therein. This work was supported in part by a NASA GSRP Fellowship and NSF grants AST-0205999 and AST-9876783.

\section{References}

Fuentes-Masip, O., et al. 2000, AJ, 120, 752

Gilbert, A. M. 2002, Ph.D. Thesis (University of California, Berkeley)

Johnson, K. E., Indebetouw, R., \& Pisano, D. J. 2003, AJ, 126, 101

Kudritzki, R. \& Puls, J. 2000, ARA\&A, 38, 613

Leitherer, C., et al. 1999, ApJS, 123, 3

Martin, C. L., Kobulnicky, H. A., \& Heckman, T. M. 2002, ApJ, 574, 663

Smith, M. G. \& Weedman, D. W. 1970, ApJ, 161, 33

Turner, J. L., et al. 2003, Nature, 423, 621.

Whitmore, B. C., \& Zhang, Q. 2002, AJ, 124, 1418

Zezas, A., Fabbiano, G., Rots, A. H., \& Murray, S. S. 2002, ApJ, 577, 710 